\title{
13 INCREASING ALL STAFF AWARENESS AND SKILLS TO PROMOTE THE CONVERSATION: A MULTI-STAFF, REGIONAL EDUCATION APPROACH
}

M Matthiesen', K Wrigley' ${ }^{1}$ Stories To Change, Cic; ${ }^{2}$ Greater Manchester and Cheshire Cancer Network

10.1136/bmjspcare-2012-000250.112

As part of the National End of Life Strategy recommendations and the Northwest Evaluation of Dying Matters Week 2010, Greater Manchester Cheshire Cancer Network aimed to provide enhanced communication skills training to all staff to achieve the following aims:

Engaging both public and professionals in end of life conversations/advance care planning.

Forging community alliances around end of life conversations and care.

GMCCN and the Conversations for Life programme delivered a nationally endorsed approach to assist staff in overcoming the fear of starting these conversations with patients/clients in order to support patient-centred care. 'Local champions' were identified to recruit staff and a series of staff development days were held and evaluated across the region. Multi-service staff attended each session, providing greater awareness of the need and benefits of early conversations, multi-disciplinary team communication and enhanced patient-centred planning. Enhanced staff attitudes and approaches were found to support local strategies around end of life, palliative care and carepathway interventions in various settings: community, care home, acute trust and those working in long-term conditions. Outcomes and evaluations to date from more than 143 participating staff and commissioners will be shared. 